\title{
Impact of Helicobacter Pylori Infection on Duodenal Microbial Community Structure and Microbial Metabolic Pathways
}

Tadashi Maeda ( $\nabla$ tadashi.ma@med.toho-u.ac.jp)

Toho University: Toho Daigaku https://orcid.org/0000-0002-9475-8909

Hiroaki Zai

Toho Daigaku

Yuto Fukui

Toho Daigaku

Yoshifumi Kato

Kyushu Daigaku

Eri Kumade

Toho University: Toho Daigaku

Toshiyasu Watanabe

Toho University: Toho Daigaku

Norihiro Furusyo

Kyushu Daigaku

Hitoshi Nakajima

Toho Daigaku

Kazuho Arai

Toho Daigaku

Yoshikazu Ishii

Toho Daigaku

kazuhiro tateda

Toho Daigaku

Yoshihisa Urita

Toho Daigaku

\section{Research}

Keywords: Helicobacter pylori, duodenal microbiota, LEfSe, KEGG, microbial metabolic pathway

Posted Date: February 4th, 2021

DOl: https://doi.org/10.21203/rs.3.rs-166718/v1 
License: (c) (i) This work is licensed under a Creative Commons Attribution 4.0 International License. Read Full License 


\section{Abstract}

\section{Background}

Recent reports suggest that Helicobacter pylori infection may be related to the onset of certain diseases. However, the $H$. pylori-related factors that play a role in the etiology of these diseases have not been fully elucidated. This study aimed to elucidate the impact of $\mathrm{H}$. pylori infection on the structure of commensal duodenal microbiota and their biofunctions.

\section{Methods}

Forty-seven (20 male, 27 female) subjects who underwent gastric cancer screening were enrolled. Duodenal fluid samples were aspirated from the descending duodenum and analyzed by 16S rRNA gene sequencing.

\section{Results}

Thirteen subjects were positive for H. pylori, while thirty-four were negative. We observed 1404 bacterial operational taxonomic units from 23 phyla and 253 genera. In the H. pylori-positive group, we observed higher abundances of Proteobacteria and lower abundances of Actinobacteria and TM7 than that in the H. pylori-negative group. The abundances of 10 genera differed significantly between the $H$. pyloripositive and -negative groups. Microbiota features in the H. pylori-positive group was significantly influenced by 12 taxa primarily belonging to Gammaproteobacteria. Microbial functional annotation collated using the Kyoto Encyclopedia of Genes and Genomes Orthology database showed that 12 microbial metabolic pathways (Synthesis and degradation of ketone bodies, Tryptophan metabolism, Nglycan biosynthesis, Ether lipid metabolism, Lipoarabinomannan (LAM) biosynthesis, Linoleic acid metabolism, alpha-Linolenic acid metabolism, Biotin metabolism, Carotenoid biosynthesis, Phenylpropanoid biosynthesis, Biosynthesis of siderophore group nonribosomal peptides, Biosynthesis of terpenoids and steroids) were significantly affected by $H$. pylori infection.

\section{Conclusions}

H. pylori infection disrupted the normal bacterial communities in the duodenum and changed the biofunctions of the commensal microbiota, primarily by upregulating specific metabolic pathways. This alteration may be related to the onset mechanisms of the diseases suspected of being related to $\mathrm{H}$. pylori infection.

\section{Background}

The presence of Helicobacter pylori infection results in the development of chronic gastritis or peptic ulcer and is also related to the development of certain gastric cancers. Recent epidemiological findings suggest that the prevalence of cardiovascular disease, haematological disease, neurodegenerative disease, liver disease, and metabolic syndrome is high in patients with H. pylori infection [1-5]. However, 
the causal relationships between the pathogenesis factors in $H$. pylori infections and various other extra gastroduodenal diseases remain unknown $[2,6,7]$.

The theory that the bioactivities of commensal gut microbiota markedly influence host biofunction has attracted considerable attention, and studies aimed at the elucidation of the pathophysiology of various diseases have been conducted. Additionally, the duodenum plays a key role in the establishment of crosstalk between the gut and the central nervous system because the release of brain-gut hormones and neurotransmitters in the small intestine, including the duodenum, is regulated by food stimuli, dietary behaviours, and the information arising from the intraluminal environment. These hormones regulate widespread biofunctions, such as metabolism, biosynthesis, feeding behaviour, and gastrointestinal functions [8-10].

Schulz et al. (2016) have reported that $H$. pylori infection alters the duodenal microbiota based on evidence obtained from the analysis of reverse-transcribed 16S rRNA and that the same results were derived based on the investigation of duodenal biopsies and aspirates [11]. These findings suggest that alteration in the duodenal microbiota induced by $H$. pylori infection is related to the onset of various other gastroduodenal diseases. This is because certain degradation products of digestion, attributed to duodenal microbial biofunction, serve as chemical effectors for host biofunctions [12, 13].

Based on the above-mentioned information, we aimed to elucidate the impact of $H$. pylori infection on the structure of the commensal duodenal microbiota and their biofunctions using conventional microbial taxonomic diversity analyses and the novel linear discriminant analysis (LDA) effect size (LEfSe) algorithm method to discover metagenomic biomarkers that could explain differences among microbial communities [14]. We also performed metagenomic functional predictions using the Kyoto Encyclopedia of Genes and Genomes (KEGG) database to infer the microbial genetic features associated with biological functions and metabolic pathways [15-17], and this approach enabled the extraction of specific genetic information related to microbial biofunction from a chaotic metagenomic bin.

\section{Results}

All 47 samples provided sufficient gene information for analysis. Thirty-four and thirteen samples were $H$. pylori-negative and $H$. pylori-positive, respectively. Considering the Kimura-Takemoto classification of endoscopic atrophy, 30 of the 34 participants (88.2\%) in the H. pylori-negative group presented with CO (non-atrophy) $-\mathrm{C} 2$ atrophy, and most of the remaining 4 participants presented with a history of $H$. pylori eradication therapy. In contrast, $\mathrm{C} 0-\mathrm{C} 2$ atrophy was observed in 5 of the 13 participants (38.5\%) in the $H$. pylori-positive group (Additional File 1). These results indicated that gastric acid secretion was decreased in the H. pylori-positive group. Analysis using MiSeq sequencing produced 3,312,084 reads with a mean of $70,470 \pm 14,833$ sequences per sample. We identified 1,404 bacterial operational taxonomy units (OTUs) from 23 phyla and 253 genera. The numbers of bacterial OTUs per participant were $263.86 \pm$ 78.46 and $284.53 \pm 67.20$ in the $H$. pylori-negative and $H$. pylori-positive groups, respectively. These 
analyses were based on a rarefied table determined from 8,804 gene reads per sample. Sequence data were submitted to DDBJ under the accession number PRJDB8081.

\section{Influence of $\mathrm{H}$. pylori on microbial diversity}

Neither a- nor $\beta$-diversity markedly differed between the H. pylori-positive and $H$. pylori-negative groups (Figs. 2 and 3 , respectively).

\section{Influence of $\mathrm{H}$. pylori on bacterial community structures}

Figure 4 illustrates the bacterial community structures at the phylum level. The relative mean abundances of Actinobacteria and TM7 were significantly higher in the $H$. pylori-negative compared to the $H$. pyloripositive group. In contrast, the abundance of Proteobacteria was significantly higher in the $\mathrm{H}$. pyloripositive group, and Acidobacteria and Planctomycetes were evident only in the H. pylori-negative group. The relative mean abundances of 14 (out of 18 ) detected phyla did not remarkably differ between the $H$. pylori-negative and $\mathrm{H}$. pylori-positive groups. The data for the other four phyla were invalid for statistical analysis (Additional File 2).

The relative mean abundances of 10 genera significantly differed between the $H$. pylori-negative and $H$. pylori-positive groups (Table 1). Only the relative abundance of Neisseria was markedly higher in the $H$. pylori-positive group, and the relative abundances of the other nine genera (Rothia, \{unknown order\} TM73, Leptotrichia, \{unknown genus\} Lachnospiraceae, Megasphaera, \{unknown genus\} F16, Moryella, Filifactor, and Paludibacter) were markedly higher in the H. pylori-negative group. Furthermore, 188 and 143 genera were detected in the H. pylori-negative and H. pylori-positive groups, respectively. These differences were attributed to the different microbial community structures observed within each group. Specifically, 60 and 15 genera were found only in the H. pylori-negative and H. pylori-positive groups, respectively. These results suggest that the duodenal microbial environment can maintain greater microbial diversity without $H$. pylori compared to that maintained with its presence. Helicobacter was detected only in the H. pylori-positive group, with an abundance of $2.79 \% \pm 6.82 \%$ (Additional File 3 ).

\section{Influence of $\mathrm{H}$. pylori on biologically relevant features}

The LDA score derived from LEfSe analyses indicated that 12 taxa markedly influenced the biological features of the duodenal microbiota in the H. pylori-positive group (Fig. 5). These 12 taxa comprised three phyla (Streptophyta, Cyanobacteria, and TG5), one class (Gammaproteobacteria), one order (Pasteurellales), one family (Enterobacteriaceae), three genera (Pseudomonas, Moraxella, and Actinobacillus), and three species (Streptococcus porcinus, Haemophilus segnis, and Paenibacillus durum) of bacteria. Six of these taxa belonged to the class Gammaproteobacteria (phylum Proteobacteria): Pasteurellales, Pseudomonas, Moraxella, Actinobacillus, H. segnis, and Enterobacteriaceae. Moraxella and $H$. segnis are notable intraoral bacteria. Two taxa, $S$. porcinus and $P$. durum, belonged to the class Bacilli (belonging to Firmicutes). These results suggest that 


\section{Influence of $\mathrm{H}$. pylori on duodenal microbial biofunctions}

Among 327 investigated KEGG pathways (Additional File 4), 163 were metabolic. The KEGG Orthology (ko)-abundance of 12 of these metabolic pathways markedly differed in the presence or absence of $H$. pylori infection (Table 2), and the ko-abundance of 9 of these 12 was remarkably greater in the $\mathrm{H}$. pyloripositive group. This suggests that $H$. pylori generally promotes metabolic functional activities in duodenal microbes. The remaining pathways were associated with genetic information processing $(\mathrm{n}=$ $16)$, environmental information processing $(n=21)$, cellular processes $(n=19)$, organismal systems $(n=$ 48), and human diseases $(n=60)$. Among these 164 pathways, 18 markedly differed between the individuals with and without $H$. pylori. However, it was not possible to infer whether these pathways were functional for microbial biofunctions and whether the pathways affected host biofunctions.

\section{Discussion}

Although $H$. pylori infection has long been suspected to result in the development of cardiovascular disease, haematologic disease, and metabolic syndrome, the roles of commensal microbiota in these diseases have remained unknown. The present study demonstrated that $H$. pylori infection markedly influenced the relative abundances of three phyla and ten genera in the duodenal microbiota, and that the altered duodenal microbiota was characterised by increased Neisseria abundance and an enhanced impact of Gammaproteobacteria. The abundance of multiple commensal microbial metabolic pathways was also considerably altered. These results suggested that $H$. pylorialtered the production of microbial metabolites, which might impact host biofunctions. Many studies have investigated the community structure and diversity of the gut microbiota before and after administration of therapeutic agents for various diseases. Although many comparative studies on the gut microbiota have associated differences in the gut microbiota with the development and pathophysiology of certain diseases, the results remain inadequate, especially, for determination of the factors originating from altered microbiota that are substantial etiological effectors.

The present $\alpha$ - and $\beta$-diversity analyses showed no significant differences between $H$. pylori-positive and H. pylori-negative groups (Figs. 2 and 3). However, these results do not necessarily indicate that an identical abundance or representation of bacterial species exists in each group. In fact, the duodenal bacterial community structures differed at the phylum level between the two groups, with greater abundance of Proteobacteria and lower abundances of Actinobacteria and TM7 (Saccharibacteria) in the H. pylori-positive group. The greater abundance of Proteobacteria is a predictable natural consequence because $H$. pylori belongs to Epsilonproteobacteria, which is a subclass of Proteobacteria. However, the bacterial community structure at the genus level and LEfSe results suggest that $H$. pylori infection altered the microbial features by increasing the abundance of Neisseria and by enhancing the impact of Gammaproteobacteria in the duodenum (Table 1 and Fig. 5). The increase in Neisseria in the duodenum 
is probably related to the gastric acid output attributed to atrophic gastritis induced by $H$. pylori. Intraoral indigenous bacteria in the genus Neisseria are not generally highly pathogenic, except for Neisseria gonorrhoeae and Neisseria meningitidis, which respectively cause gonorrhoea and meningitis [18]. However, excessive Neisseria proliferation in the duodenum may be somewhat pathogenic based on the Neisseria-associated changes induced in the microbial community structure $[19,20]$. The subclass Gammaproteobacteria consists of several medically important bacteria belonging to families, such as Enterobacteriaceae, Vibrionaceae, and Pseudomonadaceae.

Many studies examining the relationships between H. pylori infection and the development of extragastric diseases have identified increased short-chain fatty acid (SCFA) production induced by the proliferation of Bacteroidetes as a potentially important causative factor. These SCFAs induce the release of gut hormones, such as peptide YY and glucose-like peptide-1, the activation of host metabolic pathways, the elicitation of mucosal immune response, and trigger inflammation [21-23]. The present study found that $H$. pylori did not markedly change the abundance of Bacteroidetes in the duodenum (Fig. 4). The LDA scores also indicated that taxa belonging to Bacteroidetes did not significantly impact duodenal microbial features (Fig. 5). These findings were consistent with those of previous analyses conducted using duodenal aspirates [8]. Although the notion that increased SCFA production leads to the development of various diseases is attractive, SCFAs are generated primarily as a result of the fermentation of nonhost-digestible dietary fibres by the colonic microbiota. Therefore, other factors associated with upper gastrointestinal microbial functions warrant further exploration.

The KEGG pathway analysis provided substantial data for investigation of microbial function and showed that 12 bacterial metabolic pathways were affected by the presence or absence of $H$. pylori infection. Two pathways that were upregulated in the H. pylori-positive group, synthesis and degradation of ketone bodies (ko00072) and ether lipid metabolism (ko00565), are important for fatty acid degradation, butyrate and acetic acid synthesis, and the production of phosphocholine or seminolipid, which functions in the maintenance of mucosal integrity and immunohomeostasis [24, 25].

Notably, the ko-abundance of the tryptophan metabolism pathway (ko00380) was markedly greater in the H. pylori-positive group, suggesting that an abnormal tryptophan provision from the intestine impaired serotonin production. Serotonin is a paracrine messenger expressed primarily in enterochromaffin cells and enteric neurons. Various effects of serotonin on brain-gut interactions are exerted mainly through serotonin receptors on peripheral intrinsic (IPAN) and extrinsic (EPAN) primary afferent neurons [26, 27]. Therefore, disruption of the continuous and subtle tryptophan provision attributed to the presence of altered microbiota may result in the development of nerve circuit disorders involving serotonin release. This information would help to clarify the causal relationships between $H$. pylori infection, the duodenal microbiota, and the pathophysiology of functional dyspepsia [28-30]. Additionally, issues related to serotonin production may alter local serotonin concentrations in portal blood, which can also affect the gut-liver axis [31]. 
The pathways of linoleic (ko00591) and a-linolenic (ko0592) acid metabolism were also upregulated in the H. pylori-positive group (Table 2). Such an upregulation may cause an imbalance between the levels of the $\omega-3$ and $\omega-6$ fatty acids and affect the arachidonic acid cascade that is associated with the occurrence of inflammation [32]. The biotin metabolic pathway (ko00780) was also upregulated in the $H$. pylori-positive group. Bacteria synthesise biotin, which is an indispensable essential cofactor for fatty acid biosynthesis. The production of vitamin A may also be affected by $H$. pylori, because the biosynthetic pathway of the vitamin A precursor, carotenoid (ko00906), was upregulated in the H. pyloripositive group [33, 34]. The phenylpropanoid biosynthesis pathway (ko00940) was also upregulated significantly in this group. However, the physiological significance of this upregulation in humans cannot be easily determined, because the roles of metabolites (chavicol, eugenol, lignin, and so on) originating from this pathway have not been fully elucidated. The terpenoid and steroid biosynthesis pathway (ko01062) was upregulated in the H. pylori-positive group. This may extensively affect host functions, because terpenoids are steroid precursors and closely related to cytochrome P450 that functions as an oxidase in terpenoid biosynthesis $[35,36]$.

Yap et al. (2017) found 45 upregulated, and 551 downregulated serum metabolites 18 months after $H$. pylori eradication [37]. The affected metabolites were mapped to various biochemical pathways, including tryptophan metabolism, biosynthesis of unsaturated fatty acids, and linoleic acid metabolism. Although it has not been ascertained whether these alterations affect host biofunctions, our findings confirmed that the metabolomic products reported by Yap et al. originated from microbial metabolic pathways affected by H. pylori infection.

The present study also has several study limitations and issues that need to be addressed in the future. First, we could not exclude the possibility that few subjects in the H. pylori-negative group might have already experienced remarkable changes in the structure and biofunctions of their commensal duodenal microbiota due to a previous history of $H$. pylori infections. To eliminate this concern, it is necessary to analyse the changes in the microbial features before and after $H$. pylori eradication therapy in the same subject.

Furthermore, we could not completely evaluate the effect of gastric acid on the duodenal microbiota because we did not quantify gastric acid secretion. The extent of gastric mucosal atrophy caused by $H$. pylori infection is dependent on various factors, such as the age, duration of infection, differences between individual immune responses, and number of bacteria. The gastric acid output depends on the extent of gastric mucosal atrophy, and the extent of atrophic gastritis is closely related to a history of $H$. pylori infection [38]. In fact, the endoscopic findings in this study indicated that an extended atrophic change was likely to be observed in the H. pylori-positive group. Additionally, the duodenal microbiota might be affected by a decrease in the gastric acid output.

Another limitation of this study is that contamination with gastric microbiota could not be completely excluded because of the sampling method. A concern has been raised that aspirate samples include only floating microbiota, a phenomenon which may exhibit a less remarkable association with host 
biofunctions, and that the microbiota present in biopsy samples (mucosa-associated microbial community structure) actually inhabit the mucosa, a phenomenon that may be considered to expound microbiota-host associations [39, 40]. Our findings suggest that microbial metabolite production may fluctuate depending on changes in the commensal duodenal microbiota, and that this phenomenon may impact host biofunctions. These mechanisms may not be affected by aspects, such as the duodenal juice or mucosa, which are regions that are inhabited by microbes because microbial metabolites act as chemical effectors.

\section{Conclusions}

In conclusion, $H$. pylori infection changed aspects of the microbiota in the descending part of the duodenum. This dysbiosis altered the commensal microbial biofunctions, characterised mainly by the upregulation of microbial metabolic pathways, which might affect host biofunctions. The gut microbiota can be regarded as an independent organ within the gut lumen, and an investigation of biofunctions originating from this "commensal bacterial organ" would help to elucidate the aetiology of various diseases.

\section{Methods}

\section{Participants}

This study included 20 male and 27 female patients (mean age: $58.8 \pm 11.3$ years) who were screened for gastric cancer. We obtained information from all participants about treatment with gastric acid inhibitors, antibiotics, and a medical history of H. pylori eradication (Additional File 1). Patients treated with antibiotics within 4 weeks before sampling were excluded. The study protocol (Fig. 1) was implemented under the approval of the Ethics Committee of Toho University School of Medicine (authorization number: A16080), in accordance with current good clinical practice and the Declaration of Helsinki (2013). All participants provided written informed consent to participate before enrollment.

\section{Patient and public involvement}

This design of this study proceeded without public involvement. Patient involvement was restricted to sample collection at the time of enrollment. Patients were neither consulted to interpret the results, nor were invited to contribute to writing or editing this article.

\section{Collection of duodenal fluid samples and oesophagogastroduodenoscopy}

Duodenal fluid samples were collected from the descending part of the duodenum using a PW-2L-1 fluororesin tube (Olympus, Tokyo, Japan) under standard video endoscopy with the Olympus GIF-XQ260 or GIF-XP260N video gastroscope (Olympus, Tokyo, Japan). The tube was sterilised and changed for each patient. Duodenal fluid was aspirated immediately after injecting $5 \mathrm{~mL}$ of saline into the descending 
part of the duodenum, and the aspirate was immediately cryopreserved at $-80^{\circ} \mathrm{C}$. One certified endoscopist $(\mathrm{HZ})$ conducted all endoscopic procedures and sampling to avoid generation of bias. The endoscopic findings were recorded simultaneously, and the extent of atrophic gastritis was evaluated in accordance with the Kimura-Takemoto classification for endoscopic atrophy [41].

\section{Extraction of genomic DNA}

Genomic DNA (gDNA) was extracted from duodenal fluid using the PowerFecal DNA Isolation Kits (Mo Bio Laboratories, Inc., Carlsbad, CA, USA), as per the manufacturer's instructions.

\section{Identification of H. pylori}

We performed conventional nested polymerase chain reactions (PCR) using the extracted gDNA samples [42] and then sequenced amplicons to confirm the presence of $H$. pylori. The participants were then assigned to groups based on the presence or absence of $H$. pylori.

\section{Sequencing}

Sequencing libraries were prepared for analysis using the Illumina MiSeq platform (Illumina, San Diego, CA, USA). The V3 and V4 regions of the 16S rRNA gene were targeted using the primer pair 341F (5'CCTACGGGNGGCWGCAG-3') and 806R (5'-GACTACHVGGGTATCTAATCC-3') [43], and a 16S rRNA gene library was prepared for sequencing as per the manufacturer's instructions (https://support.illumina.com/documents/documentation/chemistry_documentation/16s/16smetagenomic-library-prep-guide-15044223-b.pdf). The PCR amplicons were purified using the Wizard SV Gel and PCR Clean-Up System (Promega, St. Louis, MO, USA), then sequenced and quantified using the MiSeq system [44].

\section{Bioinformatics and statistical analyses}

The sequences were processed and clustered into OTU with $97 \%$ identity using the Greengenes database (version 13.5) as the reference [45]. We evaluated bacterial diversity by calculating $\alpha$ - and $\beta$-diversity from rarefied OTU tables. The a-diversity was determined using richness (based on the number of OTUs) and evenness (appraised by the Shannon diversity index) [46, 47]. Differences in diversity indices between the $H$. pylori-positive and H. pylori-negative groups were compared using Welch's $t$-tests. The $\beta$-diversity was evaluated, based on the OTU table [48], as the unweighted UniFrac distance, which can be used to distinguish dissimilarities between the microbial profiles of two samples. The $\beta$-diversity results were analysed via permutational multivariate analyses of variance (PERMANOVA) using the CLC Genomics Workbench 10.0.1 and CLC Microbial Genomics Module 2.5 (Qiagen, Hilden, Germany). The relative abundances of phyla and genera in the H. pylori-positive and $H$. pylori-negative groups were compared based on the unrarefied OTU tables using Welch's $t$-tests.

The LEfSe algorithm can identify genomic taxa with a relative abundance that markedly differs between groups. We computed LEfSe using the Galaxy web application and workflow framework 
(https://huttenhower.sph.harvard.edu/galaxy/) to support high-dimensional class comparisons with a focus on metagenomic analysis.

Biofunctions of the duodenal microbiota were inferred via metagenomic functional annotation. The OTU abundance table was uploaded to the Piphillin server (https://piphillin.secondgenome.com/) with the KEGG Orthology (ko) database as the reference genomic database [49]. Thereafter, KEGG pathways were identified based on the gene information in the OTU, and this approach enabled interpretations of highlevel biofunctions of the microbiota.

The results of KEGG pathway analysis are identified by 'ko' followed by a five-digit number and include quantitative information. Each genome copy number can represent an abundance of genome content in each OTU that is expressed as ko-abundance. Each ko-abundance corresponds to the abundance of a specific KEGG pathway and quantitatively represents microbial biofunction characteristics. Here, we extensively investigated KEGG pathways classified under metabolism because the gut microbiota could be regarded as an independent organ in the gut lumen with metabolic functions that produces various metabolites [13]. Each ko-abundance was compared between the $H$. pylori-positive and $H$. pylori-negative groups using the Mann-Whitney $U$ tests. All values with $\mathrm{P}<0.05$ were considered statistically significant.

\section{Declarations}

Ethics approval and consent to participate and Consent for publication: The study protocol (Fig. 1) was implemented under the approval of the Ethics Committee of Toho University School of Medicine (authorization number: A16080), in accordance with current good clinical practice and the Declaration of Helsinki (2013). All participants provided written informed consent to participate before enrollment.

Availability of data and materials: Data are available in a public, open access repository. Sequence data are available from DDBJ (https://www.ddbj.nig.ac.jp/) under the accession number PRJDB8081.

Competing interests: The authors declare no competing interest.

Funding: This study was supported by JSPS KAKENHI Grant Number JP20K17003 (Grant-in-Aid for EarlyCareer Scientists), Japan Hospital General Medical Society, and Toho University School of Medicine Research Project Grant Numbers 18-21.

Author contributions: TM, $\mathrm{HZ}$, and YF contributed to the study design, analysis, and interpretation of the data. TM, HZ, YF, and EK drafted the manuscript. TM, HZ, YK, TW, and HN collected samples and identified suitable subjects. $H Z, N F, H N, K A, Y I, K T$, and $Y U$ supervised the study procedures. $T M, H Z$, and YF conducted laboratory experiments and analyses. TM, HZ, and YF conducted bioinformatics and statistical analyses. All authors read and approved the final version of manuscript. TM and $\mathrm{HZ}$ contributed equally to the present study.

Acknowledgments: The authors are grateful to the staff in the Department of Microbiology and Infectious Diseases, Toho University School of Medicine, and to all the participants. The authors extend their 
gratitude toward Professor Hidekazu Suzuki, Department of Gastroenterology, Tokai University School of Medicine for providing guidance. We thank Editage (www.editage.com) for the English language editing services provided.

\section{References}

1. Gasbarrini A, Fox J, Gasbarrini G. Helicobacter pylori and other Helicobacter chronic infections and extragastric diseases. Eur J Gastroenterol Hepatol. 2000;12(9):1057-60. 10.1097/00042737200012090-00020, PMID: 11386215

2. Ražuka-Ebela D, Giupponi B, Franceschi F. Helicobacter pylori and extragastric diseases. Helicobacter. 2018;23(Suppl 1):e12520. 10.1111/hel.12520, PMID: 30203590

3. Zendehdel A, Roham M. Biological evidence of the relationship between Helicobacter pylori and associated extragastric diseases. J Cell Biochem. 2019;120(8):12128-40. 10.1002/jcb.28681, PMID: 30977160

4. Peng W, Yi P, Yang J, Xu P, Wang Y, Zhang Z, Huang S, Wang Z, Zhang C. Association of gut microbiota composition and function with a senescence-accelerated mouse model of Alzheimer's disease using 16S rRNA gene and metagenomic sequencing analysis. Aging (Albany, NY). 2018;10(12):4054-65. 10.18632/aging.101693, PMID: 30562162

5. Kim MS, Kim Y, Choi H, Kim W, Park S, Lee D, Kim DK, Kim HJ, Choi H, Hyun DW, Lee JY. Transfer of a healthy microbiota reduces amyloid and tau pathology in an Alzheimer's disease animal model. Gut. 2020;69(2):283-94. 10.1136/gutjnl-2018-317431, PMID: 31471351

6. Waskito LA, Salama NR, Yamaoka Y. Pathogenesis of Helicobacter pylori infection. Helicobacter. 2018;23(Suppl 1):e12516. 10.1111/hel.12516, PMID: 30203582

7. Schulz C, Schütte K, Malfertheiner P. Helicobacter pylori and other gastric microbiota in gastroduodenal pathologies. Dig Dis. 2016;34(3):210-6. 10.1159/000443353, PMID: 27028228

8. Holtmann G, Shah A, Morrison M. Pathophysiology of functional gastrointestinal disorders: a holistic overview. Dig Dis. 2017;35(Suppl 1):5-13. 10.1159/000485409, PMID: 29421808

9. Jung HK, Talley NJ. Role of the duodenum in the pathogenesis of functional dyspepsia: a paradigm shift. J Neurogastroenterol Motil. 2018;24(3):345-54. 10.5056/jnm18060, PMID: 29791992

10. Zhong L, et al. Dyspepsia and the microbiome: time to focus on the small intestine. Gut. 2017;66(6):1168-9. 10.1136/gutjnl-2016-312574, PMID: 27489239

11. Schulz, C. et al. The active bacterial assemblages of the upper $\mathrm{Gl}$ tract in individuals with and without Helicobacter infection. Gut. 2018;67(2);216-25. 10.1136/gutjnl-2016-312904, PMID: 27920199

12. Obata $Y$, Pachnis V. The effect of microbiota and the immune system on the development and organization of the enteric nervous system. Gastroenterology. 2016;151(5):836-44.

10.1053/j.gastro.2016.07.044, PMID: 27521479 
13. Shanahan F, van Sinderen D, O'Toole PW, Stanton C. Feeding the microbiota: transducer of nutrient signals for the host. Gut. 2017;66(9):1709-17. 10.1136/gutjnl-2017-313872, PMID: 28663354

14. Segata N, et al. Metagenomic biomarker discovery and explanation. Genome Biol. 2011;12(6):R60. 10.1186/gb-2011-12-6-r60, PMID: 21702898

15. Kanehisa M, Furumichi M, Tanabe M, Sato Y, Morishima K. KEGG: new perspectives on genomes, pathways, diseases and drugs. Nucleic Acids Res. 2017;45(D1):D353-61. 10.1093/nar/gkw1092, PMID: 27899662

16. Kanehisa M, Goto S. KEGG: Kyoto encyclopedia of genes and genomes. Nucleic Acids Res. 2000;28(1):27-30. 10.1093/nar/28.1.27, PMID: 10592173

17. Kanehisa M, Sato Y, Furumichi M, Morishima K, Tanabe M. New approach for understanding genome variations in KEGG. Nucleic Acids Res. 2019;47(D1):D590-5. 10.1093/nar/gky962, PMID: 30321428

18. Liu G, Tang CM, Exley RM. Non-pathogenic Neisseria: members of an abundant, multi-habitat, diverse genus. Microbiology. 2015;161(7):1297-312. 10.1099/mic.0.000086, PMID: 25814039

19. Clemence MEA, Maiden MCJ, Harrison OB. Characterization of capsule genes in non-pathogenic Neisseria species. Microb Genom. 2018;4(9):e000208. 10.1099/mgen.0.000208, PMID: 30074474

20. Donati $C$, et al. Uncovering oral Neisseria tropism and persistence using metagenomic sequencing. Nat Microbiol. 2016;1(7):16070. 10.1038/nmicrobiol.2016.70, PMID: 27572971

21. Castaño-Rodríguez N, Mitchell HM, Kaakoush NO. NAFLD, Helicobacter species and the intestinal microbiome. Best Pract Res Clin Gastroenterol. 2017;31(6):657-68. 10.1016/j.bpg.2017.09.008, PMID: 29566909

22. Tilg H, Moschen AR. Microbiota and diabetes: an evolving relationship. Gut. 2014;63(9):1513-21. 10.1136/gutjnl-2014-306928, PMID: 24833634

23. van de Wouw M, Schellekens H, Dinan TG, Cryan JF. Microbiota-gut-brain axis: modulator of host metabolism and appetite. J Nutr. 2017;147(5):727-45. 10.3945/jn.116.240481, PMID: 28356427

24. Gonçalves P, Araújo JR, Di Santo JP. A cross-talk between microbiota-derived short-chain fatty acids and the most mucosal immune system regulates intestinal homeostasis and inflammatory bowel disease. Inflam Bowel Dis. 2018;24(3):558-72. 10.1093/ibd/izx029, PMID: 29462379

25. Hiippala, K. et al. The potential of gut commensals in reinforcing intestinal barrier function and alleviating inflammation. Nutrients. 2018;10(8):988. 10.3390/nu10080988, PMID: 30060606

26. Gershon MD, Tack J. The serotonin signaling system: from basic understanding to drug development for functional GI disorders. Gastroenterology. 2007;132(1):397-414. 10.1053/j.gastro.2006.11.002, PMID: 17241888

27. Vanner S, Macnaughton WK. Submucosal secretomotor and vasodilator reflexes. Neurogastroenterol Motil. 2004;16(Suppl 1):39-43. 10.1111/j.1743-3150.2004.00473.x, PMID: 15066003

28. De Vadder $F$, et al. Gut microbiota regulates maturation of the adult enteric nervous system via enteric serotonin networks. Proc Natl Acad Sci USA. 2018:115(25);6458-63.

10.1073/pnas.1720017115, PMID: 29866843

Page 13/22 
29. Israelyan $\mathrm{N}$, et al. Effects of serotonin and slow-release 5-hydroxytryptophan on gastrointestinal motility in a mouse model of depression. Gastroenterology. 2019;157(2):507-21.e4. 10.1053/j.gastro.2019.04.022, PMID: 31071306

30. Wauters L, Talley NJ, Walker MM, Tack J, Vanuytsel T. Novel concepts in the pathophysiology and treatment of functional dyspepsia. Gut. 2020;69(3):591-600. 10.1136/gutjnl-2019-318536, PMID: 31784469

31. Choi W, et al. Serotonin signals through a gut-liver axis to regulate hepatic steatosis. Nat Commun. 208;9(1):4824. 10.1038/s41467-018-07287-7. Erratum in: PMID: 30446669

32. Cunnane SC. Problems with essential fatty acids: time for a new paradigm? Prog Lipid Res. 2003;42(6):544-68 10.1016/s0163-7827(03)00038-9, PMID: 14559071

33. Moran NE, Mohn, ES, Hason, N, Erdman, JW Jr, Johnson EJ. Intrinsic and extrinsic factors impacting absorption, metabolism, and health effects of dietary carotenoids. Adv Nutr. 2018;9(4):465-92. 10.1093/advances/nmy025, PMID: 30032230

34. Said HM. Biotin: biochemical, physiological, and clinical aspects. In Stanger, O., editor. Water Soluble Vitamins. Subcellular Biochemistry. 56. Dordrecht: Springer; 2012. p. 1-19. 10.1007/978-94-0072199-9_1, PMID: 22116691

35. Bian G, Deng Z, Liu T. Strategies for terpenoid overproduction and new terpenoid discovery. Curr Opin Biotechnol. 2017;48:234-41. 10.1016/j.copbio.2017.07.002, PMID: 28779606

36. Loke MF, et al. Metabolomics and 16S rRNA sequencing of human colorectal cancers and adjacent mucosa. PLOS ONE. 2018;13(12):e0208584. 10.1371/journal.pone.0208584, PMID: 30576312

37. Yap TW, et al. Global fecal and plasma metabolic dynamics related to Helicobacter pylori eradication. Front Microbiol. 2017;8:536. 10.3389/fmicb.2017.00536, PMID: 28424674

38. Mihara $\mathrm{M}$, et al. The role of endoscopic findings for the diagnosis of Helicobacter pylori infection: evaluation in a country with high prevalence of atrophic gastritis. Helicobacter. 1999;4(1):40-8. 10.1046/j.1523-5378.1999.09016.x, PMID: 10352086

39. Nishino K, et al. Analysis of endoscopic brush samples identified mucosa-associated dysbiosis in inflammatory bowel disease. J Gastroenterol. 2018;53(1): 95-106. 10.1007/s00535-017-1384-4, PMID: 28852861

40. Ni, J., Wu, G. D., Albenberg, L. \& Tomov, V. T. Gut microbiota and IBD: causation or correlation? Nat Rev Gastroenterol Hepatol. 2017;14(10):573-84. 10.1038/nrgastro.2017.88, PMID: 28743984

41. Kimura K, Takemoto T. An endoscopic recognition of the atrophic border and its significance in chronic gastritis. Endoscopy. 1969;1(3):87-97. 10.1055/s-0028-1098086

42. Rimbara E, Sasatsu M, Graham DY. PCR detection of Helicobacter pylori in clinical samples. In Wilks, M, editor. PCR Detection of Microbial Pathogens. Methods in Molecular Biology (Methods and Protocols). 943. Totowa, NJ: Humana Press; 2013. p. 279-287. 10.1007/978-1-60327-353-4_19, PMID: 23104297

43. Klindworth A, et al. Evaluation of general $16 \mathrm{~S}$ ribosomal RNA gene PCR primers for classical and next-generation sequencing-based diversity studies. Nucleic Acids Res. 2013;41(1):e1. 
10.1093/nar/gks808, PMID: 22933715

44. Jiang H, Barker SC, Shao R. Substantial variation in the extent of mitochondrial genome fragmentation among blood-sucking lice of mammals. Genome Biol Evol. 2013;5(7):1298-1308. 10.1093/gbe/evt094, PMID: 23781098

45. DeSantis TZ, et al. Greengenes, a chimera-checked 16S rRNA gene database and workbench compatible with ARB. Appl Environ Microbiol. 2006;72(7):5069-72. 10.1128/AEM.03006-05, PMID: 16820507

46. Kim BR, et al. Deciphering diversity indices for a better understanding of microbial communities. J Microbiol Biotechnol. 2017;27(12):2089-93. 10.4014/jmb.1709.09027, PMID: 29032640

47. Ringel $Y$, et al. High throughput sequencing reveals distinct microbial populations within the mucosal and luminal niches in healthy individuals. Gut Microbes. 2015;6(3):173-81.

10.1080/19490976.2015.1044711, PMID: 25915459

48. Lozupone C, Lladser ME, Knights D, Stombaugh J Knight R. UniFrac: an effective distance metric for microbial community comparison. ISME J. 2011;5(2):169-72. 10.1038/ismej.2010.133, PMID: 20827291

49. Iwai $S$, et al. Piphillin: improved prediction of metagenomic content by direct inference from human microbiomes. PLOS ONE. 2016;11(11):e0166104. 10.1371/journal.pone.0166104, PMID: 27820856

\section{Tables}


Table 1 Differences in the relative mean abundances of genera in Helicobacter pyloripositive and $H$. pylori-negative groups.

Relative mean abundance $(\%) \pm \mathrm{SD}$

\begin{tabular}{lccc} 
Genus & H. pylori & H. pylori & P* \\
& -negative $(\mathrm{n}=34)$ & -positive $(\mathrm{n}=13)$ & \\
\hline Neisseria & $4.76 \pm 5.93$ & $11.74 \pm 7.10$ & $<0.01$ \\
Rothia & $6.83 \pm 7.44$ & $1.81 \pm 1.36$ & $<0.001$ \\
\{Unknown Order\} TM7-3 & $2.74 \pm 3.54$ & $0.80 \pm 0.89$ & $<0.01$ \\
Leptotrichia & $2.06 \pm 1.84$ & $1.18 \pm 1.02$ & $<0.05$ \\
\{Unknown Genus\} Lachnospiraceae & $0.90 \pm 0.84$ & $0.33 \pm 0.30$ & $<0.01$ \\
Megasphaera & $0.72 \pm 0.76$ & $0.38 \pm 0.33$ & $<0.05$ \\
\{Unknown Genus\} F16 & $0.49 \pm 0.60$ & $0.21 \pm 0.23$ & $<0.05$ \\
Moryella & $0.32 \pm 0.39$ & $0.11 \pm 0.13$ & $<0.05$ \\
Filifactor & $0.28 \pm 0.45$ & $0.09 \pm 0.16$ & $<0.05$ \\
Paludibacter & $0.04 \pm 0.06$ & $0.01 \pm 0.02$ & $<0.05$ \\
\hline
\end{tabular}

Ten taxa with significant differences among 253 detected are shown. Few taxa that could not be identified at the genus level were classified at higher levels. Notations in parentheses indicate the classification level. *Calculated using Welch's $t$-tests. 
Table 2 Differences in KEGG metabolic pathways between Helicobacter pylori-positive and H. pylori-negative groups.

\begin{tabular}{clllll}
\hline & & & \multicolumn{3}{c}{ Median ko-abundance } \\
Class & $\begin{array}{c}\text { KEGG pathway } \\
\text { (ko number) }\end{array}$ & \multicolumn{1}{c}{ Pathway } & $\begin{array}{l}\text { H. pylori } \\
\text {-negative } \\
(\mathrm{n}=34)\end{array}$ & $\begin{array}{l}\text { H. pylori } \\
\text {-positive } \\
(\mathrm{n}=13)\end{array}$ & $\mathrm{p}^{\dagger}$ \\
\hline M & ko00072* & Synthesis and degradation of ketone bodies & 1335885.8 & 1823878.4 & $<0.05$ \\
M & ko00380* & Tryptophan metabolism & 2254680.9 & 2759405.6 & $<0.05$ \\
M & ko00510 & N-glycan biosynthesis & 907554.4 & 700748.0 & $<0.05$ \\
M & ko00565* & Ether lipid metabolism & 150966.1 & 394815.2 & $<0.001$ \\
M & ko00571 & Lipoarabinomannan (LAM) biosynthesis & 397200.0 & 191311.5 & $<0.05$ \\
M & ko00591* & Linoleic acid metabolism & 85148.3 & 389478.0 & $<0.001$ \\
M & ko00592* & alpha-Linolenic acid metabolism & 132544.9 & 405656.4 & $<0.001$ \\
M & ko00780* & Biotin metabolism & 15103400 & 19564468 & $<0.05$ \\
M & ko00906* & Carotenoid biosynthesis & 129310.3 & 510048.5 & $<0.01$ \\
M & ko00940* & Phenylpropanoid biosynthesis & 348411.2 & 598460.1 & $<0.01$ \\
M & ko01053 & Biosynthesis of siderophore group & 2025414.2 & 1127312.8 & $<0.01$ \\
& & nonribosomal peptides & & & $<0.01$ \\
M & ko01062* & Biosynthesis of terpenoids and steroids & 92469.4 & 428251.7 & $<$ \\
\hline
\end{tabular}

Twelve metabolic pathways that significantly differed among the 327 detected pathways. M, metabolism.

* Most abundant pathways in $H$. pylori-positive group. ${ }^{\dagger}$ Calculated using the Mann-Whitney $U$ tests.

\section{Figures}




\section{7 subjects \\ I}

Duodenal fluids collection under endoscopic examination

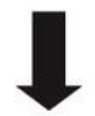

DNA extraction and 16S rRNA sequence analysis

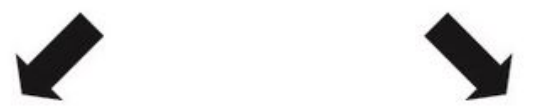

H. pylori negative $(\mathrm{n}=34) \quad H$. pylori positive $(\mathrm{n}=13)$

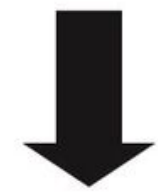

Bioinformatics analyses

a-diversity

- $\beta$-diversity

- Bacterial community structures

LEfSe analysis

-KEGG mapping

Figure 1

Study outline. Duodenal microbial bioinformatics data were compared between Helicobacter pyloripositive and H. pylori-negative groups. KEGG, Kyoto Encyclopedia of Genes and Genomes; LEfSe, linear discriminant analysis effect size. 
A

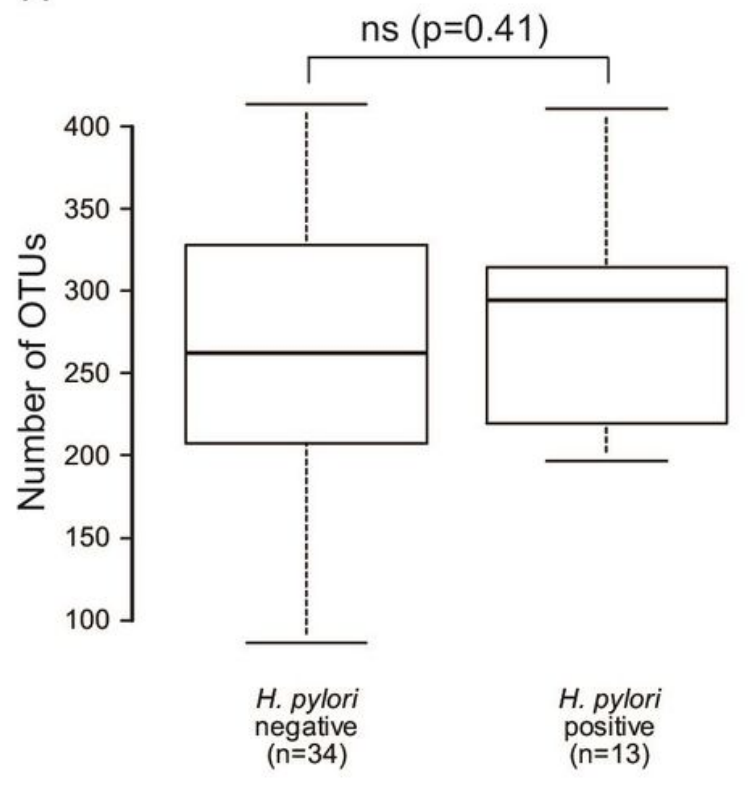

B

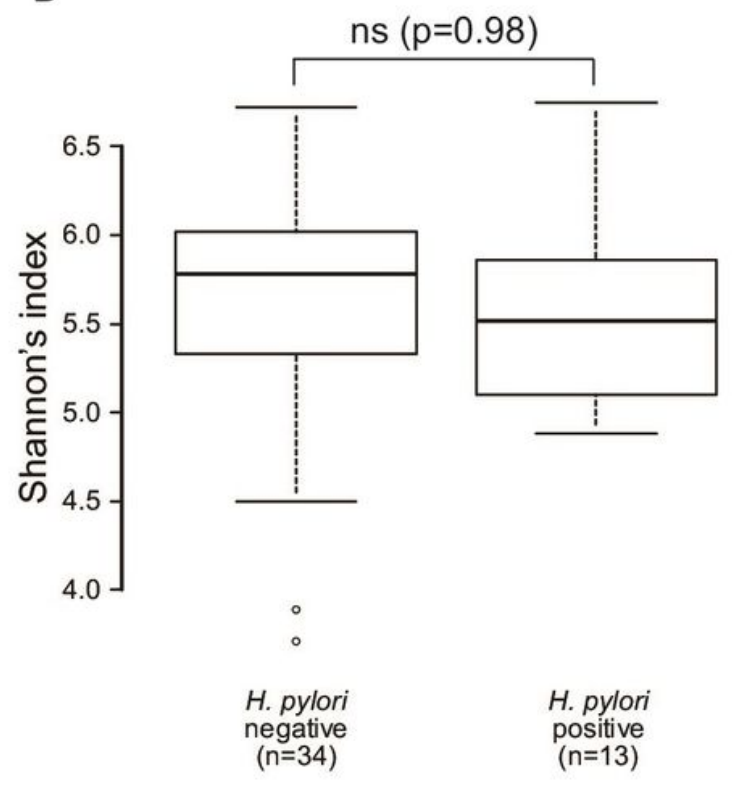

\section{Figure 2}

Comparison of the a-diversity of the duodenal microbiota between Helicobacter pylori-positive and $\mathrm{H}$. pylori-negative groups. (A) Richness, (B) Evenness. OTU: operational taxonomic units. ns: nonspecific. P values were calculated using Welch's t-tests. 


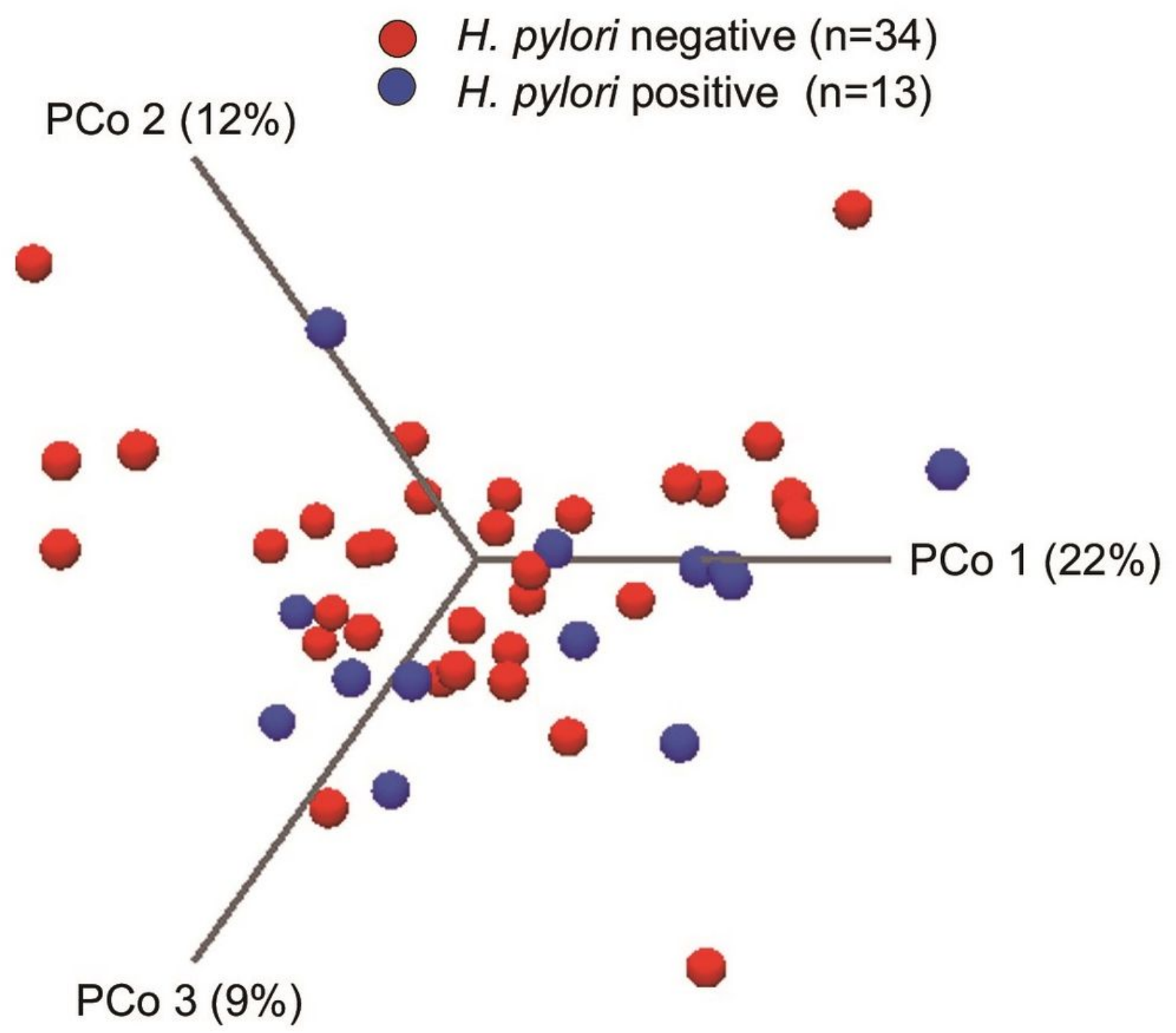

Figure 3

Principal coordinate analysis plots illustrating bacterial $\beta$-diversity data for all samples. Microbial profiles were not significantly dissimilar between Helicobacter pylori-positive and $\mathrm{H}$. pylori-negative groups. Unweighted UniFrac distance: $p=0.09$. 


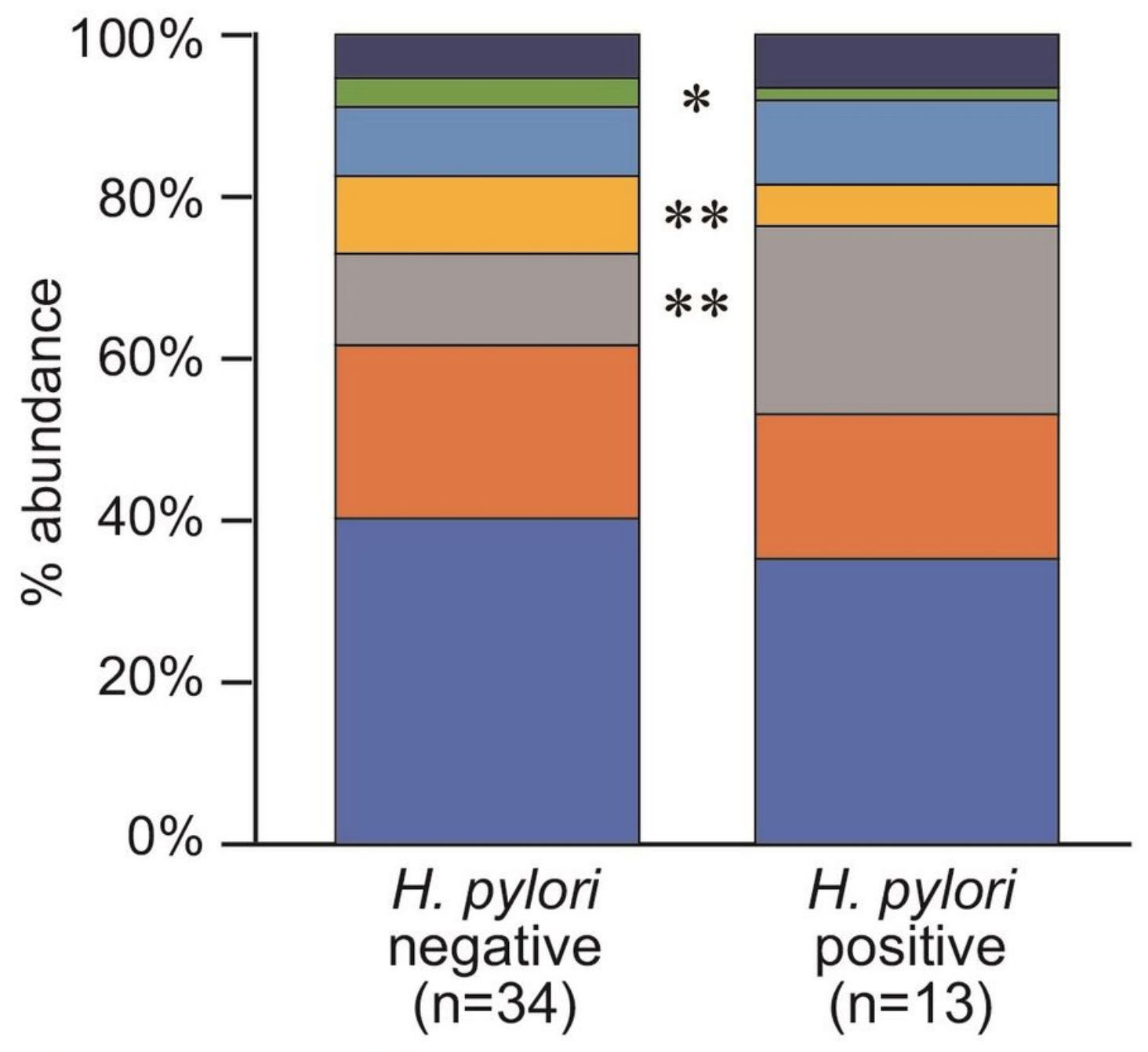

a Firmicutes Bacteroidetes Proteobacteria Actinobacteria $\square$ Fusobacteria $\square$ TM7 $\square$ others

Figure 4

Differences in the bacterial community structures at the phylum level in Helicobacter pylori-positive and H. pylori-negative groups. ${ }^{*} \mathrm{P}<0.05$ and ${ }^{\star *} \mathrm{P}<0.01$ (Welch's t-tests). 
A

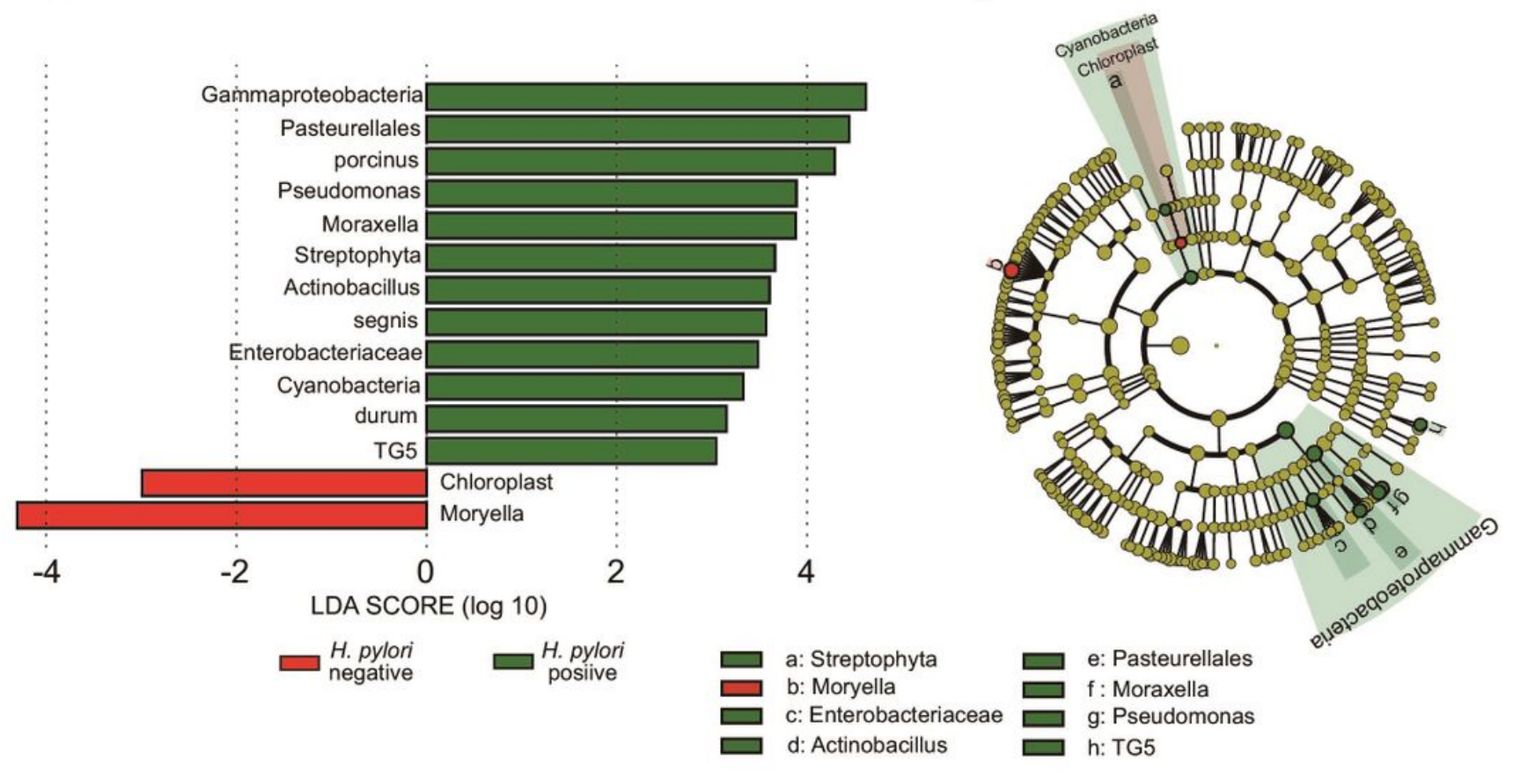

\section{Figure 5}

LEfSe analysis of differences in biologically relevant features between Helicobacter pylori-positive and $\mathrm{H}$. pylori-negative groups. Blue and red: $\mathrm{H}$. pylori-positive and H. pylori-negative, respectively. (A) Rank of effect size of each taxa. (B) Taxonomic cladogram considering hierarchy and systematic closeness.

\section{Supplementary Files}

This is a list of supplementary files associated with this preprint. Click to download.

- Suppllnformation1SubjectsinformationRevise.docx

- Suppllnformation2phylarelativeabundance.docx

- Suppllnformation3genusrelativeabundance.docx

- SupplInformation4KEGGPathway.docx 